ICACCG2020 30-31 July, 2020, Ansal University, Gurgaon, India

\title{
CONVERTING SOUND ENERGY TO ELECTRICITY
}

\author{
Satvik Sawhney ${ }^{1}$, Yash Sharma ${ }^{2}$, Bhavika Parashar ${ }^{3}$, Manimala $^{4}$, Sherry Verma ${ }^{5}$ \\ E-Mail Id: ${ }^{1}$ satviksawhney.btech18@ ansaluniversity.edu.in, ${ }^{2}$ yashsharma.btech18@ ansaluniversity.edu.in, \\ ${ }^{3}$ bhavikaparashar.btech18@ansaluniversity.edu.in, ${ }^{4}$ sherryverma@ansaluniversity.edu.in, \\ ${ }^{5}$ manimala@ansaluniversity.edu.in \\ School of Engineering \& Technology, Ansal University, Gurgaon, Haryana, India
}

Abstract-Noise Pollution is an everyday reality of a developing economy. This project seeks to convert the decibel levels of the unwanted noise to useful energy, serving 2 purposes: Working as a sustainable energy source that stores the electrical energy for future use and also indicating the noise levels in an area using a microphone and Arduino. The sound vibrations cause waves of pressure which get translated into electrical energy with the help of Piezoelectric sensor. This energy passes through a bridge rectifier and a capacitor and is finally stored in a Lithium ion battery for future use while also displaying the decibel levels (dB) and verifying it on an app called "Sound Meter", achieved with the help of Arduino. This is a sustainable source of energy and is highly cost effective making the product affordable to the masses.

This low cost device can be securely put in places encountering high level of sound. Airports, Industries, traffic intersections will provide the arena for this device to produce energy as well as serve as an indicator of noise pollution levels.

Urban arears, hospitals etc. can use this device to manage the sound pollution by tacking the data obtained. The applications of this product are endless as it serves as an inexpensive decibel-meter \& a power bank. The product can easily be made handy to masses and corporations alike.

Keywords: Arduino, Arduino IDE, Microphone, Piezoelectric sensors, Decibel Level, Sound Meter App, Voltage Regulator, Capacitors, Diodes, Lithium ion battery.

\section{INTRODUCTION}

Electricity is a secondary source of energy that is it is neither a renewable nor a non-renewable resource. It is obtained by primary source of energy such as wind energy, natural gas, solar energy, coal etc. In India the electricity sector is largely operated by fossil fuels. India holds the third rank in production as well as consumption of electricity. According to featured data of 2019-2020 fiscal year 72\% of countries electricity was generated with the help of coal. India's electricity power consumption is expected to increase by 10 percent by annually over next 10-15 years.

So as the requirement of electricity manifold in coming decades, we will be discussing a new dimension that is how with the help sound energy electrical energy can be generated.

\section{LITERATURE REVIEW}

Sound is a mechanical energy and travels in the form of waves. Sound energy propagates in form of longitudinal wave in medium like liquid and gases whereas in solid medium its waves travel both in longitudinal as well as transverse manner.

Most of the sound around us is unpleasant and undesirable i.e. sound waves are present in the form of noise. Noise pollution is invisible danger. Noise pollution impacts millions of people on a daily basis. Sounds above the levels of 85 decibels are harmful for humans as well as animals lining around. Humans exposed to loud noise can develop diseases such as high blood pressure, sleep disturbances, stress and heart disease. [1]

Animals use sound for a variety of reasons, including to navigate, find food, attack mates, and avoid predators. Noise pollution makes it difficult for them to accomplish these tasks, which affects their ability survive. The major cause of rising levels of noise pollution in most megalopolis is due to lack of urban planning and bazillion running vehicles on the roads. [1]

This form of energy which is present in abundance can be channelize to a beneficial source energy using suitable and appropriate technology. Sound is a mechanical energy and can be converted to electrical energy as per the law of thermodynamics.

Piezoelectricity is the ability of the material to develop electric charge in response to the applied mechanical stress (direct piezoelectric effect) and vice-versa (inverse piezoelectric effect). Presently known materials that consist piezoelectrical properties are, naturally occurring Berlinite, Quartz, Cane sugar, Rochelle, Topaz, Tourmaline etc., man-made crystals are Gallium orthophosphate $(\mathrm{GaPO})$, Langasite $\left(\mathrm{La}_{3} \mathrm{Ga}_{5} \mathrm{SiO}_{14}\right)$. Artificial piezoelectric ceramics include lead zirconate titante, lead titanate, potassium niobate, sodium niobate, zinc oxide etc. There were certain polymers that also possess piezoelectric properties. They are polyvinlydene fluoride (PVDF) and its copolymers like polyvinyldene fluoride-trifluoro ethylene (PVDF-TrFE), polyvinyldene fluoride hexafluoro propylene, odd Nylons, Polyvinyl chloride (PVC), lead zinc niobate-lead titante (PZN-PT), lead magnesium titanate (PMN-sPT) single crystals, etc. Piezoelectric materials are crucial and necessary elements for conversion of sound energies to electricity. [2] 
The equation for the resonant frequency of the cantilever type piezoelectric $-\mathrm{f}_{\mathrm{r}}=\mathrm{w} / 2 \pi=1 / 2 \pi\left[\mathrm{k} / \mathrm{m}_{\mathrm{e}}\right]^{1 / 2}, \mathrm{f}_{\mathrm{r}}$ is the resonant frequency, $\mathrm{w}=$ angular frequency, $\mathrm{k}=$ spring constant, $\mathrm{m}_{\mathrm{e}}=\mathrm{effective}$ mass of cantilever. Certain single crystal materials exhibit the following phenomenon: when the crystal is mechanically strained, (here sound energy) or when the crystal is deformed by the application of an external stress, electric charges appear on the crystal surfaces; and when the direction of the strain reverses, the polarity of the electric charge is reversed. This is called the direct piezoelectric effect, and the crystals that exhibit it are classed as piezoelectric crystal. [3]

$\mathrm{f}_{\mathrm{n}}=\mathrm{v}^{2} \mathrm{n} / 2 \pi \mathrm{L}^{2}\left[\mathrm{E}_{\mathrm{O}} / \mathrm{m}\right], \mathrm{f}_{\mathrm{n}}$ is the $\mathrm{n}^{\text {th }}$ mode resonant frequency, $\mathrm{v}_{\mathrm{n}}$ is the $\mathrm{n}^{\text {th }}$ mode eigen value, $\mathrm{L}=$ length of the cantilever and $\mathrm{m}$ the mass per unit length of the cantilever.[3]

The inverse piezoelectric effect refers to the deformation in these materials like PZT etc. that results from the application of an electric field. So it could be seen that when the sound energy is applied to the piezoelectric material it creates strain in the crystal then it reverses it and the strain is converted into electric energy. This direct piezoelectric effect property of a piezoelectric material could be used for making the device to convert sound energy to electric energy. [3]

\section{PIEZO ELECTRIC SENSOR}

Piezo electric sensor works on the principal of piezoelectric effect. The sensor is a transducer but also encompass properties of actuator. piezoelectric sensors work by applying mechanical energy to a crystal in the following steps:

$>$ A piezoelectric crystal is placed between two metal plates which are normally in a perfect balance (even if they're not symmetrically arranged) and does not conduct any electric current.

$>$ Mechanical stress or force are applied on the material by the metal plates, which forces the electric charges within the crystal out of balance. Excess negative and positive charges appear on opposite sides of the crystal face.

$>$ The metal plate collects these charges, which can be used to produce a voltage and send an electrical current through a circuit transforming to piezoelectricity. [4]

Therefore, we can convert sound energy to electrical energy by the use of piezoelectric material.

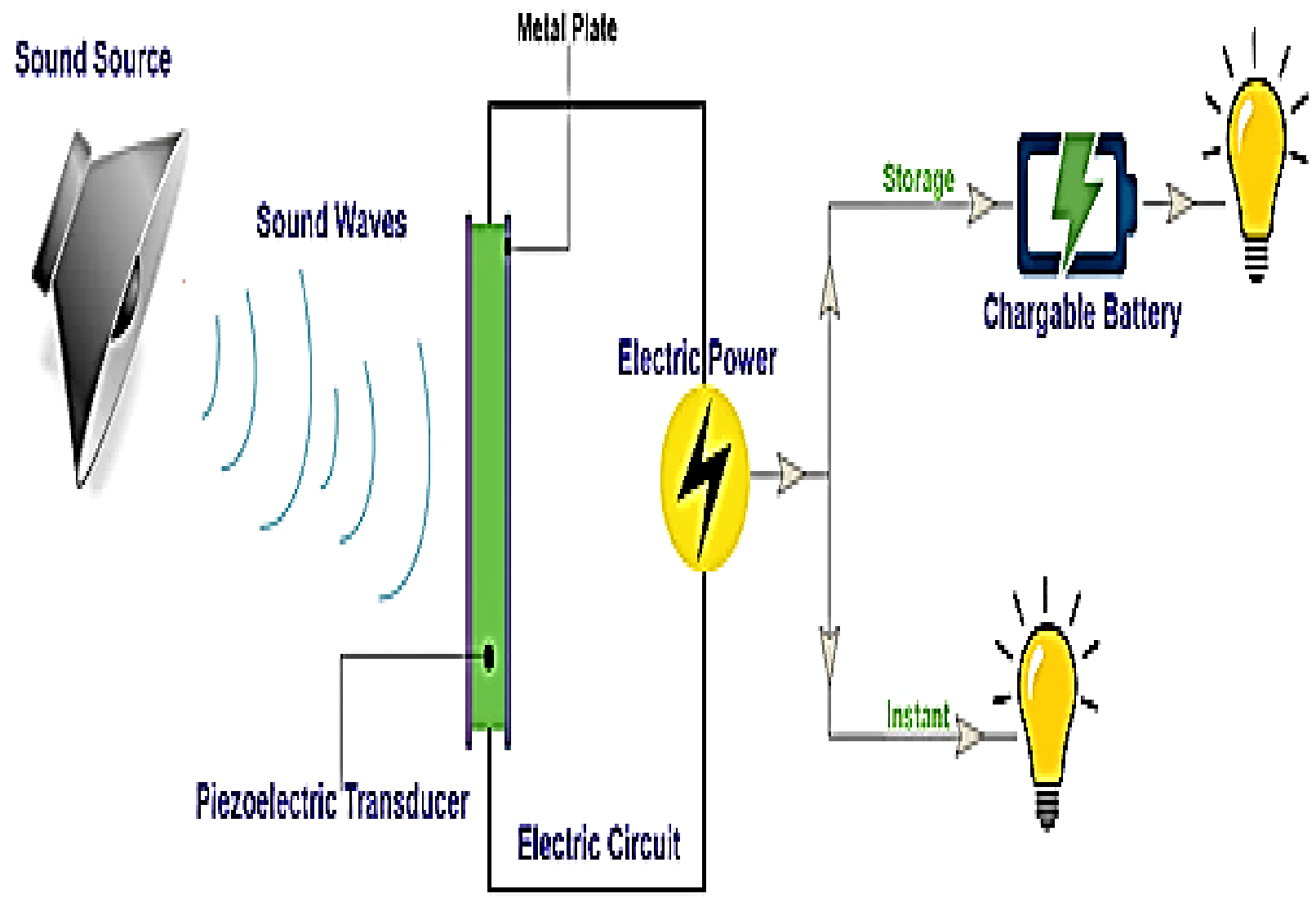

Fig. 3.1 Basic Operation of the System

\section{PROPERTIES OF PIEZOELECTRIC MATERIAL}

Different crystals having distinct materials show the following unique properties: if the crystal glass experiences an external force, electrical charges appear on the surface of the crystal; The polarity of electric charge is inverted when the direction of tension is inverted. The crystals appearing on this are known to be piezoelectric crystal. This also referred to as direct piezoelectric effect. 
ICACCG2020 30-31 July, 2020, Ansal University, Gurgaon, India

International Journal of Technical Research \& Science (Special Issue)

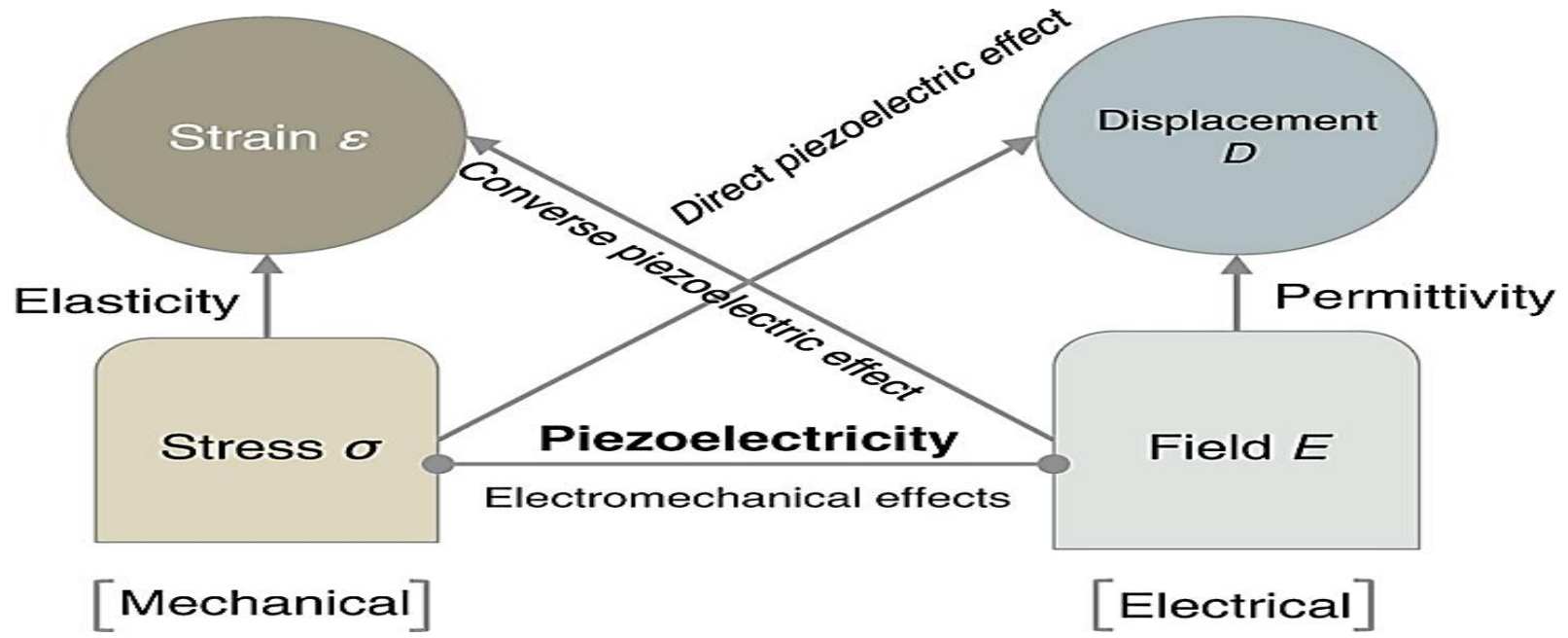

Fig. 4.1 Diagram Explaining Piezoelectric Effect

On the contrary, when a piezoelectric crystal is placed in Dynamic field, or the weight applied by an external Means on their faces, the glass shows tension, that is Glass dimensions. As the direction of electric field inverts, reversed distortion is obtained. Thus a phenomena called piezoelectric effect occurs.
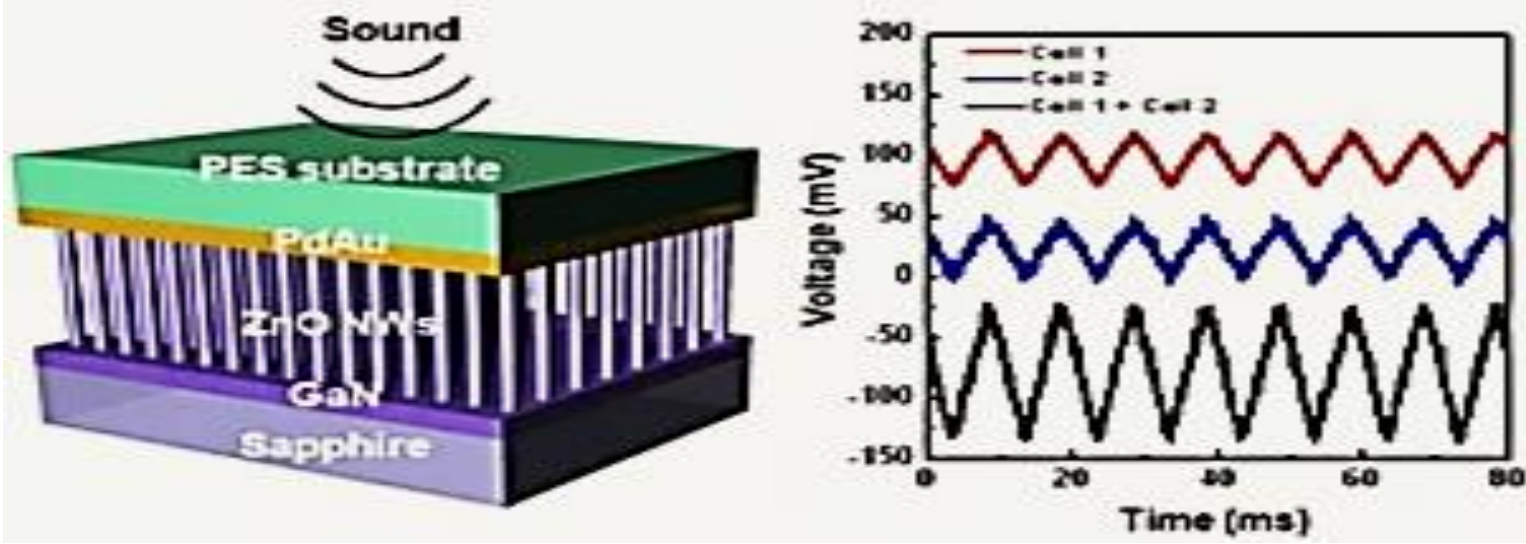

Fig. 4.2 Inverting Direction of Force

So, you can see this when the sound energy applying piezoelectric material creates tension in the crystal then turns it over and becomes electric energy. This is a direct piezoelectric effect of piezoelectric materials which is used to make a device for converting sound energy into electrical energy. The piezoelectric converter (SRB) - it is a device that can be made using piezoelectric materials whose purpose is to use the sound wave around it to cause pressure / stress. Due to this pressure, it causes oscillation in the piezoelectric crystal and this will create disturbance in their atoms which makes the electric charge flow on the face of the crystal structure, thus, sound energy is converted to electric current. Piezoelectric material converts mechanical stress into electrical energy. Therefore, this sound energy is used to carry out Various functions when converted into useful electrical energy.
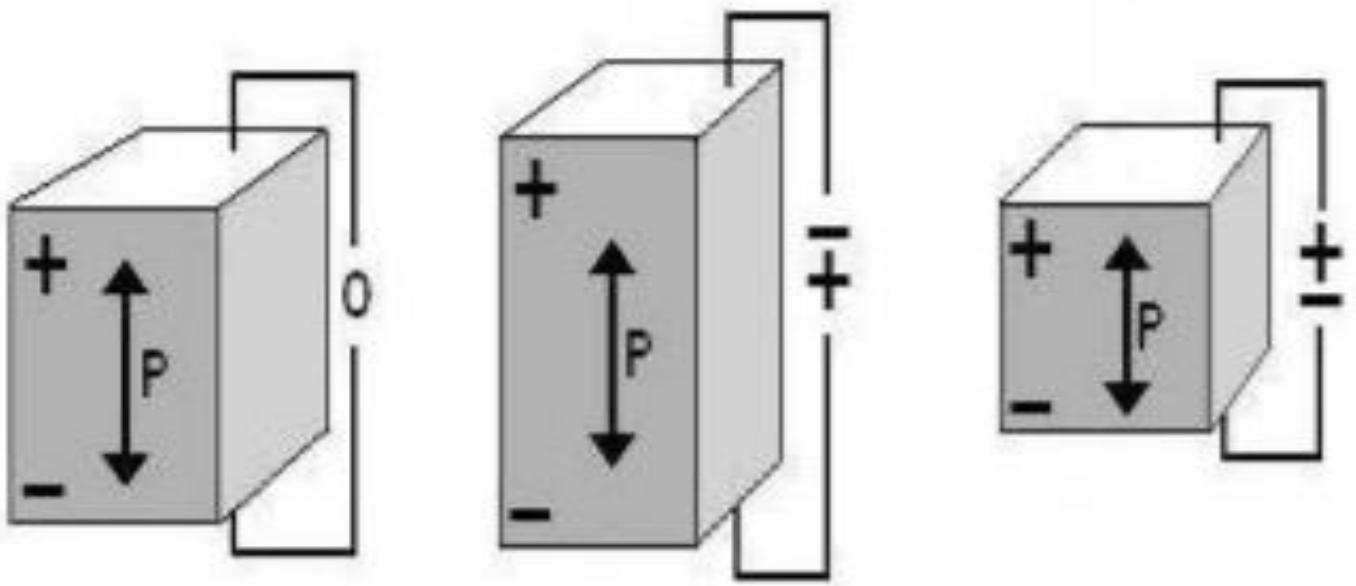

Fig 4.3 Voltage Time Graph 
ICACCG2020 30-31 July, 2020, Ansal University, Gurgaon, India

International Journal of Technical Research \& Science (Special Issue)

\section{ALTERNATE METHODS}

$>$ Transverse: $-\mathrm{x}=\mathrm{dxy}$ Fyb/a

$>$ The production of charge relys on the algorithmic dimensions of the piezoelectric element. Longitudinal: $-\mathrm{Cx}=\mathrm{dxx}$ Fxn,

$>$ where $\mathrm{dxx}$ is the piezoelectric coefficient of a charge. The charge varies with the change in $\mathrm{x}$ direction.

Shear: $-\mathrm{Cx}=2 \mathrm{dxx}$ Fxn,

Only applicable if $n$ elements mechanically are in series and electrically in parallel the charges. [6]

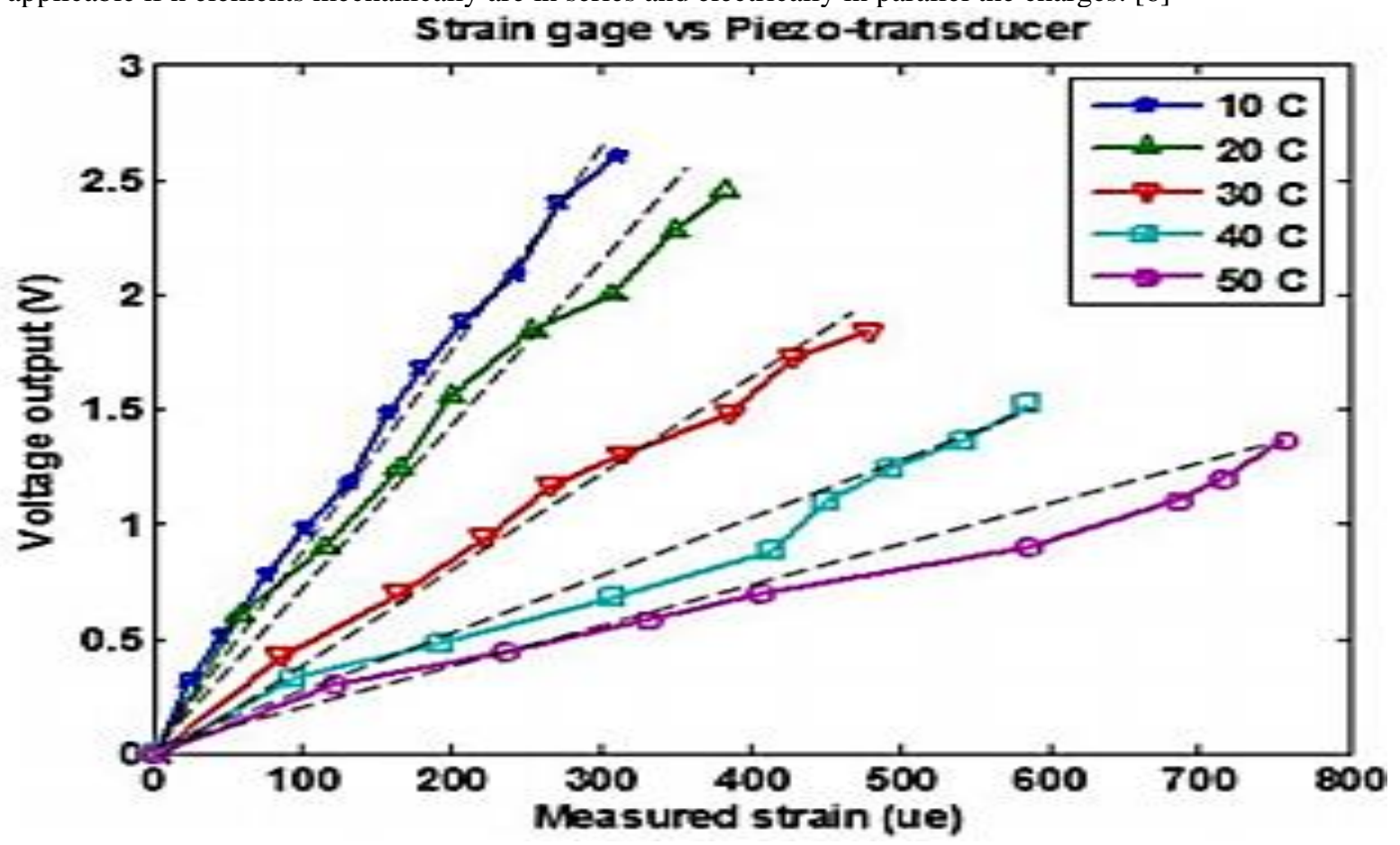

Fig. 5.1 Voltage Strain Graph

Method 1: This is another way to convert sound into thermal energy and then into electrical energy.

The waves are transmitted by the oscillation some particles / small matter in some spacious medium. When the waves of sound travel through the free space, the average particles in the medium are disturbed. This, in turn produces a disruption through the remaining medium, This results in a phenomenon being created by the various molecules within the free space / medium. This sound wave gets transformed to heat energy, thus, this results in the collision of the molecules of the medium and the resulting heat energy automatically changes to an electrical energy. A solid medium has more thermal energy / heat energy production, thus a high density material is needed in order to obtain more heat. The resultant electrical energy is obtained from this heat energy.
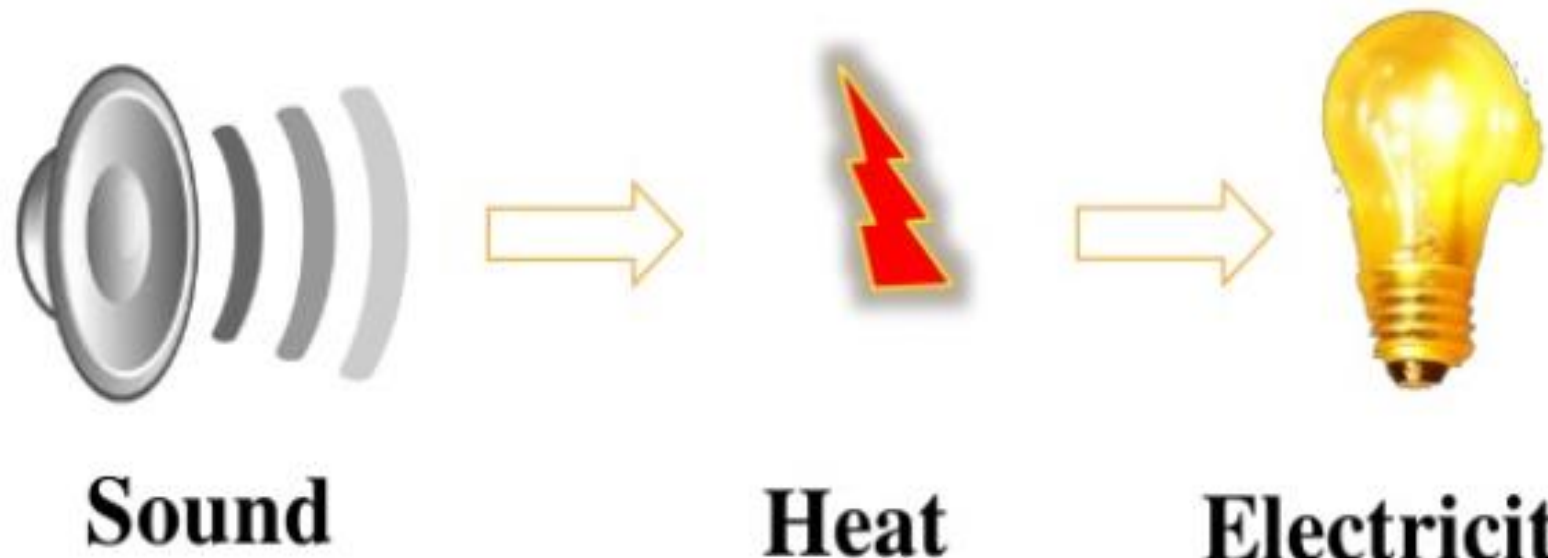

\section{Heat}

Electricity

Fig. 5.2 Sound Converted to Thermal Energy

DOI Number: https://doi.org/10.30780/specialissue-ICACCG2020/037

Paper Id: IJTRS-ICACCG2020-037

pg. 26 
ICACCG2020 30-31 July, 2020, Ansal University, Gurgaon, India

International Journal of Technical Research \& Science (Special Issue)

ISSN No.:2454-2024 (online)

However, this method results in an energy loss. This method falls short when Newton's law comes into play, stating that you can neither create, nor destroy the energy. This is a big drawback of this method.

Method 2: This method uses the following: Diaphragm, magnets and conductors. The stress that is caused by the sound waves vibrates the diaphragm. The diaphragm is connected to a conductor. There are two magnetic rods, and the diaphragm is put between them and left to vibrate;

We get some movement from EMF that is generated by the effect of conductor on the magnetic field of the magnet.

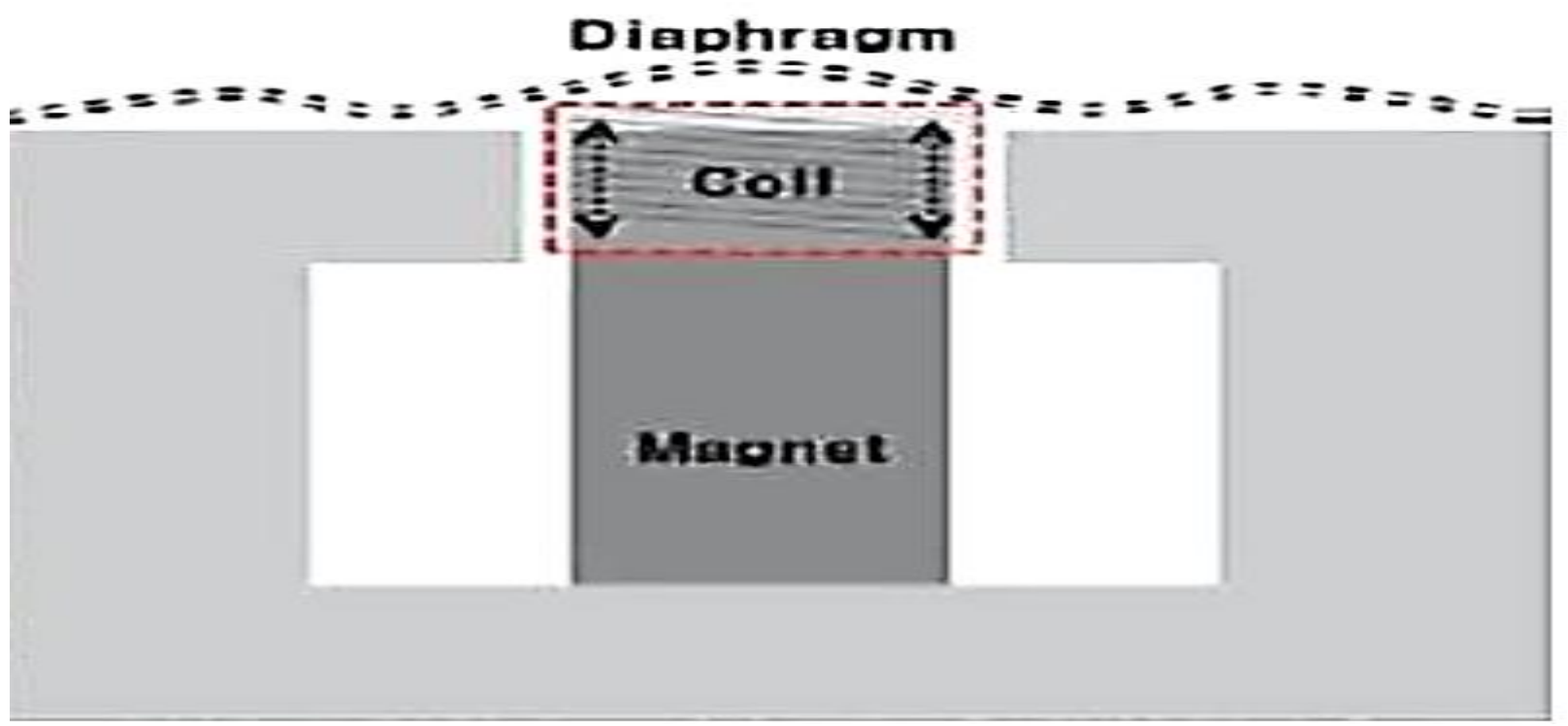

Fig. 5.3 Sound to Electricity using Diaphragm

We place a very skinny diaphragm layer that periodically vibrates because of sound waves and the pressure that they create. Next we place a conductor between the two magnetic poles. Thus, the Conductor has a magnetic flux allaround it whenever the diaphragm vibrates / oscillates.

$\mathrm{EMF}=$ Conductor's velocity * Magnetic field * conductor's length

Oscillations / vibrations increase as the frequency of vibrations increase, resulting in a good amount of energy being generated. However, like the method 2, this one also has a limitation and one that cannot be overlooked. This method needs a very loud decibel level of sound toproduce a substantial amount of energy. This method doesn't produce a huge amount of energy with average noise source, however, when coupled with a huge amount of noise that could pain the ears of humans, it shows its magic and produces huge amounts of energy / voltage, as the apparatus vibrates with more frequency than ever.

\section{USING MICROPHONE TO FIND DECIBEL LEVELS (dB)}

To achieve this, we will use a single Electret Condenser microphone with Arduino and try measuring the sound or noise pollution level in $\mathrm{dB}$ as close as possible to the actual value. We will use amplifier circuit to amplify the sound signals and feed it to Arduino, in which we would use the regression method to calculate the sound signals in dB. To check if the values obtained are correct we can use the "Sound Meter" android application.

Materials Required:

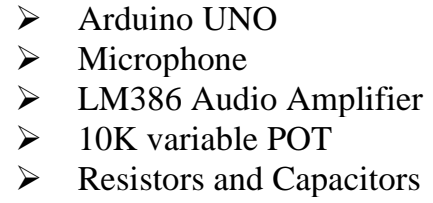

The Circuit for Arduino Sound Meter is fairly easy to make. We have used the LM386 Audio amplifier circuit to amplify the signals from the microphone and supply it to the Analog port of the Arduino. We have already built a low voltage audio amplifier circuit with LM386 IC as shown in Fig. 8. The circuit more or less remains the same. The gain of the op-amp can be set from 20 to 200 using a resistor or capacitor across pin 1 and 8 . If they are left free the gain will be set as 20 by default. For our project we will set the maximum gain possible by this circuit, so we use a capacitor of value $10 \mathrm{uF}$ between the pins 1 and 8 , note that this pin is polarity sensitive and the negative pin of capacitor should be connected to pin 8 . The entire amplifier circuit is powered by using the $5 \mathrm{~V}$ pin from Arduino.

The C2 Capacitor is used for filtering the DC noise from the Microphone. Basically when the microphone senses sound the sound waves will be converted to AC signals. This AC signal might have some DC noise coupled with it which will be filtered by this capacitor. Similarly, even after amplification a capacitor C3 is used to filter any DC noise that might have been added during amplification.

Along with amplifier we have also used two filters, the high-pass filter is formed by R5, C2 and the low-pass filter is used by the $\mathrm{C} 1$ and $\mathrm{R} 2$. These filters are designed to allow frequency only from $8 \mathrm{~Hz}$ to $10 \mathrm{KHz}$, since the low pass- 
ICACCG2020 30-31 July, 2020, Ansal University, Gurgaon, India

International Journal of Technical Research \& Science (Special Issue)

ISSN No.:2454-2024 (online)

filter will filter anything below $8 \mathrm{~Hz}$ and the High Pass filter will filter anything above $15 \mathrm{KHz}$. This concludes the Amplifier circuit.

Now we get the ADC levels into the Arduino after the electricity has passes through the Amplifier circuit. We have to convert this $\mathrm{ADC}$ value to $\mathrm{dB}$.

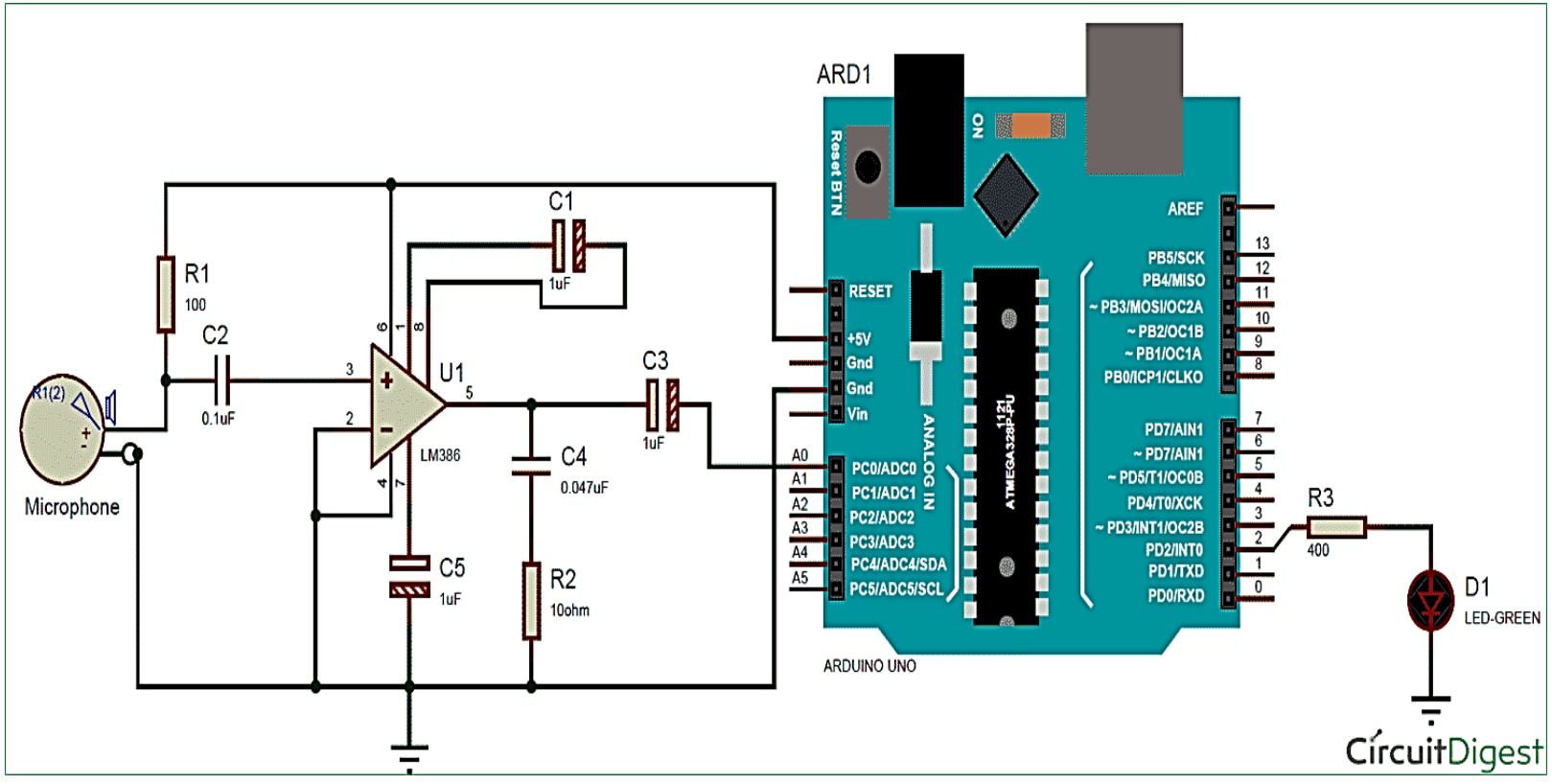

Fig. 6.1 Circuit Diagram

Table-6.1 ADC Values before Conversion to Decibel

\begin{tabular}{|c|c|}
\hline ADC value & dB \\
\hline 460 & 44 \\
\hline 480 & 47 \\
\hline 500 & 59 \\
\hline 508 & 60 \\
\hline 550 & 61 \\
\hline 600 & 63 \\
\hline 613 & 65 \\
\hline 700 & 70 \\
\hline 859 & 78 \\
\hline
\end{tabular}

6.1 Converting ADC to Decibel:

To convert ADC to Decibel, we use the "Linear Regression" method.

You get the following equation with this method:

$d B=(\mathrm{ADC}+83.2073) / 11.003$

This equation can be used to find the equivalent value of $\mathrm{dB}$ for every value of $\mathrm{ADC}$ that the Arduino measures.

Arduino Code:

adc = analogRead $(M I C)$

$d B=(\mathrm{ADC}+83.2073) / 11.003$

In these above two lines, we read the $\mathrm{ADC}$ value of pin $\mathrm{A} 0$ and convert it to $\mathrm{dB}$ using the equation that we just derived via Linear Regression.

After this, the code is to be uploaded to the Arduino.

DOI Number: https://doi.org/10.30780/specialissue-ICACCG2020/037

pg. 28

Paper Id: IJTRS-ICACCG2020-037

@ 2017, IJTRS All Right Reserved, www.ijtrs.com 
ICACCG2020 30-31 July, 2020, Ansal University, Gurgaon, India

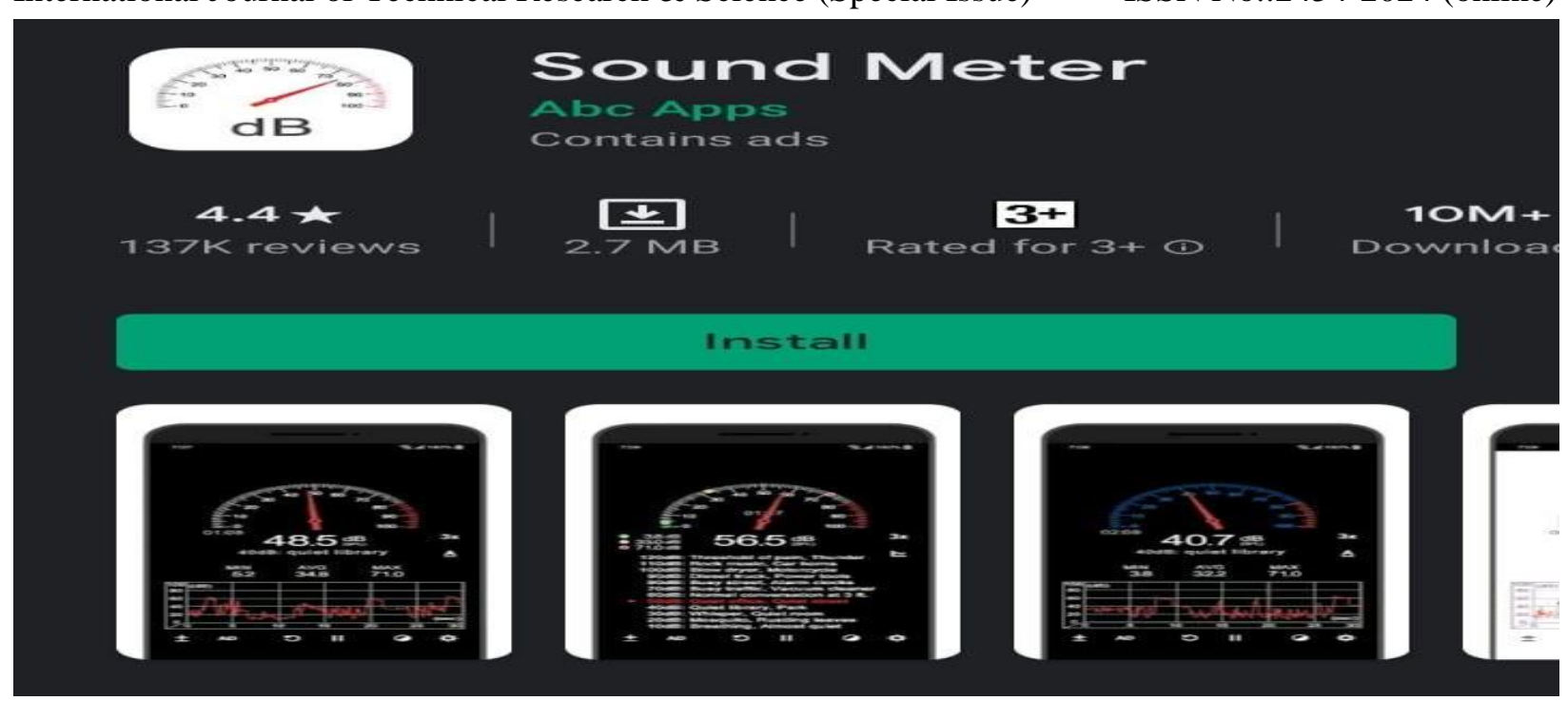

Fig. 6.2 Play Store Snapshot of Sound Meter App

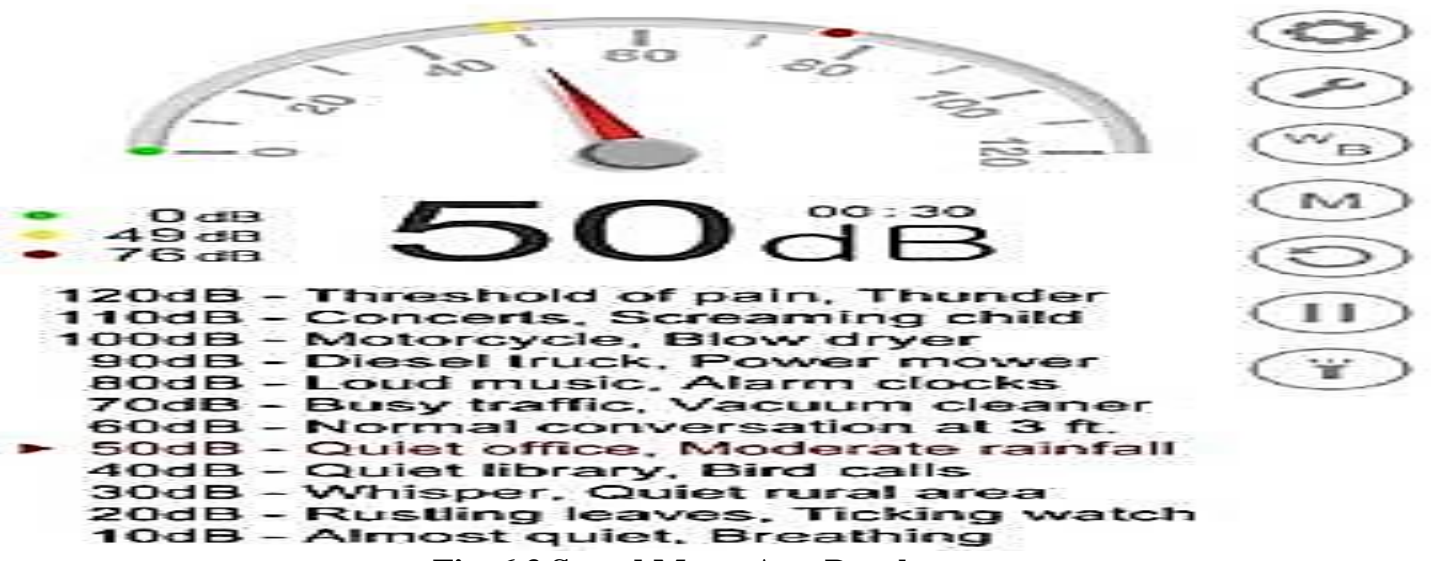

Fig. 6.3 Sound Meter App Panel

Once ready with the code and hardware, just upload the code to Arduino and open your Sound Meter App to verify the $\mathrm{dB}$ values measured by your Arduino.Please note down the value of $\mathrm{dB}$ from microphone and amplifier circuit is being displayed on the output screen of Arduino IDE and the correct $\mathrm{dB}$ values are being displayed on your mobile phone via the app, to be used as reference to verify the values on Arduino IDE.

\section{SOUND ENERGY TO ELECTRICITY USING PIEZOELECTRIC SENSORS PROCEDURE}

The Piezoelectric sensors continuously convert noise to electricity for storage into the Lithium Ion Battery. After turning on the device, it either woks as a decibel meter, or charges the Lithium Ion Battery. When the power bank / lithium ion battery is switched on, it can be used to charge / power any device connected to it.

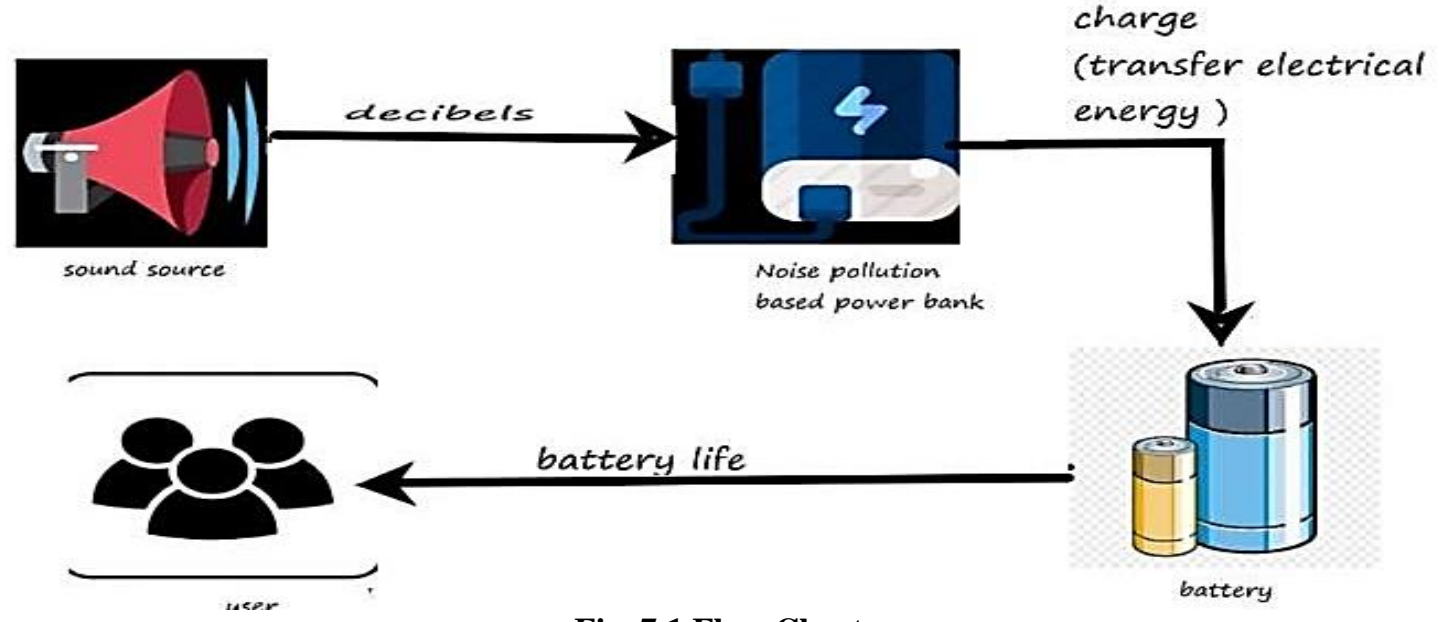

Fig. 7.1 Flow Chart

DOI Number: https://doi.org/10.30780/specialissue-ICACCG2020/037

pg. 29

Paper Id: IJTRS-ICACCG2020-037

@ 2017, IJTRS All Right Reserved, www.ijtrs.com 
In the below circuit, six Piezoelectric sensors are linked in parallel and connected to a bridge rectifier that helps the flow of voltage from alternating. The capacitor is used for storing the voltage before forwarding it to the battery for permanent storage.

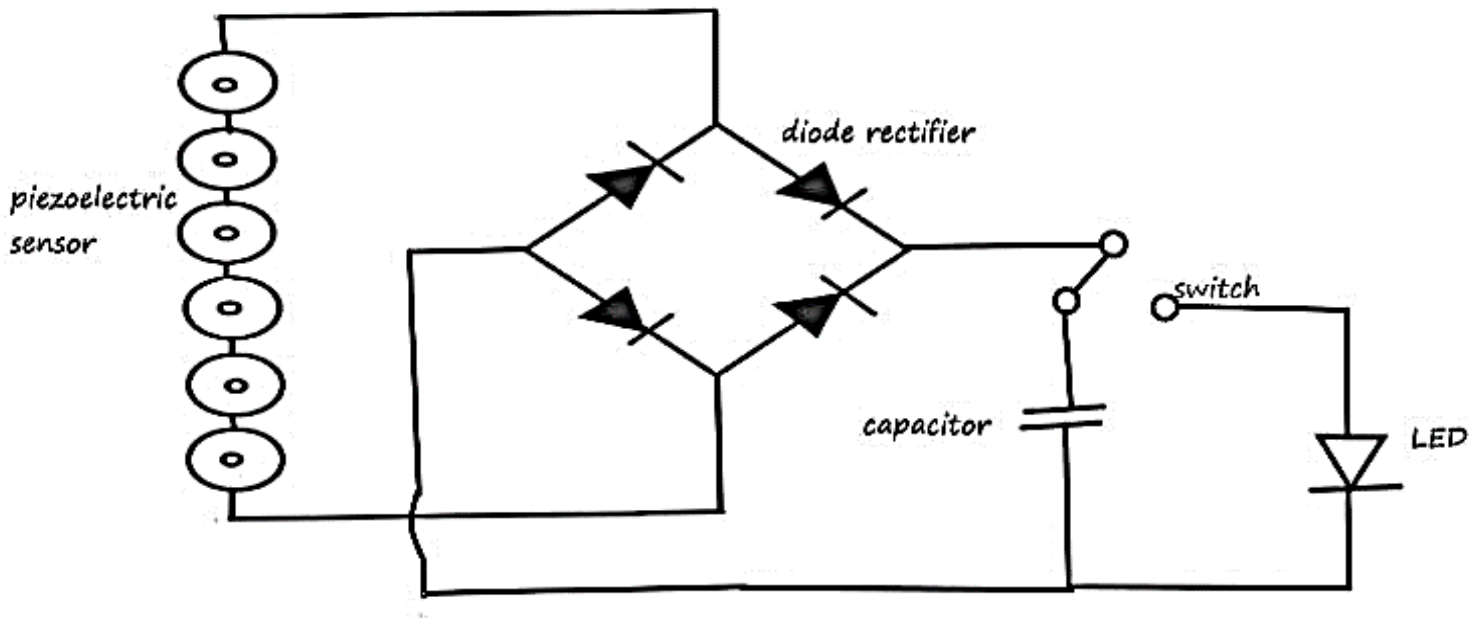

Fig. 6.5 Circuit Diagram

This entire Circuit is neatly placed in a box along with the Amplifier circuit, microphone and Arduino from before and this gives us a portable device that we can install anywhere to make electrical energy out of noise pollution and record the decibel level as per need of the user. The user can later uninstall the device, and use the lithium ion battery for charging his / her devices.

\section{SCOPE OF THE PROJECT}

This low cost device can be securely put in places encountering high levels of sound. Airports, Industries, traffic intersections will provide the arena for this device to produce energy as well as serve as an indicator of noise pollution levels.

This project can help us reduce lack of electric power around the world and thus help us in human development and reduction of carbon footprint because electric energy is one of the cleanest energies.

Urban areas, hospitals etc. can use this device to manage the sound pollution in their areas by tracking the data obtained. The applications of this product are endless as it serves as an inexpensive decibel-meter \& a power bank. The product can easily be made accessible to the masses and corporations alike

\section{CONCLUSION}

This paper aims to explain how electricity can be produced from sound waves / energy. There are several ways to convert sound energy to electrical energy such as follows:

$>$ By using an apparatus made up of a magnet and a conductor.

$>$ By converting sound energy to heat energy and finally to Electricity.

$>$ By using transducers such as piezo electric material. It can convert mechanical to electrical energy.

We discussed a new dimension to convert sound energy to electricity, which is made possible due to Piezoelectric effect. Since sound energy is generated at every part of earth surface and is part of nature it can be utilized to produce sustainable electrical energy. The production of electrical energy depends majorly on high decibel level of sound energy and sound receiving diodes. Electricity demands are expected to rise by $40 \%$ by 2040 . So, generating electricity with help of piezoelectric effect in our daily life can make a big difference.

\section{REFERENCES}

[1] Noise Pollution https://www.nationalgeographic.org/encyclopedia/noise-pollution/

[2] Piezoelectricity https://www.sciencedirect.com/topics/materials-science/piezoelectricity

[3] Tomar, Pulkit, Pavanesh Kumar, Neyaz Ali, Sandeep Kumar, Tahseen Musharraf, and Pramod Kumar. 2016. "Conversion Of Noise Pollution To Electrical Energy". Internal Journal Of Advance Research In Science And EngineeringVol. No.5 (Issue No.03).

[4] How do Piezoelectric sensors work?http://sensor-works.com/how-do-piezoelectric-sensors-work/

[5] Shalabh Rakesh Bhatnagar"Converting sound energy to electric energy" International Journal of Emerging Technology and Advanced Engineering ISSN 2250- 2459, Volume 2, Issue 10, October 2012. (Conference Paper)

[6] Measure Sound/Noise Level in dB with Microphone and Arduino https://circuitdigest.com/microcontrollerprojects/arduino-sound-level-measurement. 